\title{
Effects of Vitamin D Supplementation during the Induction and Progression of Osteoarthritis in a Rat Model
}

\author{
E. C. Castillo,,${ }^{1,2}$ M. A. Hernandez-Cueto, ${ }^{3}$ M. A. Vega-Lopez, ${ }^{1}$ C. Lavalle, ${ }^{4}$ \\ J. B. Kouri, ${ }^{1}$ and V. Ortiz-Navarrete ${ }^{2}$ \\ ${ }^{1}$ Departamento de Infectómica y Patogénesis Molecular, Centro de Investigación y de Estudios Avanzados (CINVESTAV), \\ IPN, Avenida IPN No. 2508, 07360 México, DF, Mexico \\ ${ }^{2}$ Departamento de Biomedicina Molecular, Centro de Investigación y de Estudios Avanzados (CINVESTAV), \\ IPN, Avenida IPN No. 2508, 07360 México, DF, Mexico \\ ${ }^{3}$ Unidad de Investigación en Infectología e Inmunología, Hospital de Infectología Dr. Daniel Méndez Hernández, \\ Centro Médico Nacional La Raza, IMSS, Avenida Jacarandas s/n, 02990 México, DF, Mexico \\ ${ }^{4}$ Facultad de Medicina, Universidad Nacional Autónoma de México (UNAM), Circuito Escolar s/n, 04510 México, DF, Mexico
}

Correspondence should be addressed to V. Ortiz-Navarrete, vortiz@cinvestav.mx

Received 18 April 2012; Revised 4 September 2012; Accepted 5 September 2012

Academic Editor: Angelo Antonio Izzo

Copyright ( 2012 E. C. Castillo et al. This is an open access article distributed under the Creative Commons Attribution License, which permits unrestricted use, distribution, and reproduction in any medium, provided the original work is properly cited.

\begin{abstract}
Epidemiological studies correlate low levels of vitamin D with the osteoarthritis (OA) progression. Cytokines and metalloproteases play a major role in OA promoting the inflammation and degradation of the cartilage and can be induced through the Toll-like receptor (TLR) pathway. The aim of this study was to evaluate the protective effect of vitamin D supplementation on the development of osteoarthritis (OA) through examining the genetic regulation of TLRs, cytokines, and metalloproteases in chondrocytes as well as the wideness of cartilage in rats with OA. Our results demonstrate that the signaling through TLR- 4 is a proinflammatory mechanism in osteoarthritis that drives the upregulation of MMP-3, IL- $1 \beta$, and TNF- $\alpha$ gene expression, leading to cartilage degradation and inflammation. Vitamin D supplementation had a protective effect during the onset but not during the chronic stage of $\mathrm{OA}$ in the rat model.
\end{abstract}

\section{Introduction}

It has been shown that TNF- $\alpha$ synthesis decreases in monocytes treated with vitamin $\mathrm{D}\left(1,25(\mathrm{OH})_{2} \mathrm{D}_{3}\right)$ due to the downregulation of TLR-2 and TLR-4 signaling [1]. In this context, epidemiological studies and experimentally induced animal models suggest that the status of vitamin $\mathrm{D}$ is associated with the severity of inflammatory diseases such as rheumatoid arthritis [2] and OA [3]. In particular, vitamin $\mathrm{D}$ deficiency has been positively associated with knee [4] and hip [5], OA progression [2,6], cartilage loss [7], and low bone mineral density [3]. However, it has also been shown that vitamin $\mathrm{D}$ deficiency is associated with only certain age groups $[4,6,8]$ and with certain factors, such as pain, rather than with radiographic changes $[9,10]$, while other studies have found no association between vitamin $\mathrm{D}$ and $\mathrm{OA}$ $[8,11,12]$, even in patients diagnosed with $\mathrm{OA}$ who were using vitamin D supplements [13].
Because epidemiological studies can only evaluate the effect of vitamin $\mathrm{D}$ in certain stages of $\mathrm{OA}$, we aimed to define whether vitamin $\mathrm{D}$ has a protective effect during the onset or progression of OA using a rat model. The effect of vitamin $\mathrm{D}$ was evaluated by measuring condyle wideness and the gene expression of key cytokines, MMPs, and TLRs that are involved in the development of OA.

\section{Methods}

2.1. Rats and Cartilage Samples. Male Wistar rats weighing $130-150 \mathrm{~g}$ were kept in acrylic cages with a stainless steel cover. The rats were housed in a controlled environment with a $12 \mathrm{~h}$ light/dark cycle, constant temperature $\left(22^{\circ} \mathrm{C}\right)$ and humidity (70\%), and free access to food and water. Their diet was standard chow (Formulab Diet 5008) containing 1\% calcium, $0.65 \%$ phosphorous, and $3.3 \mathrm{IU} / \mathrm{g}$ of vitamin $\mathrm{D}_{3}$. 


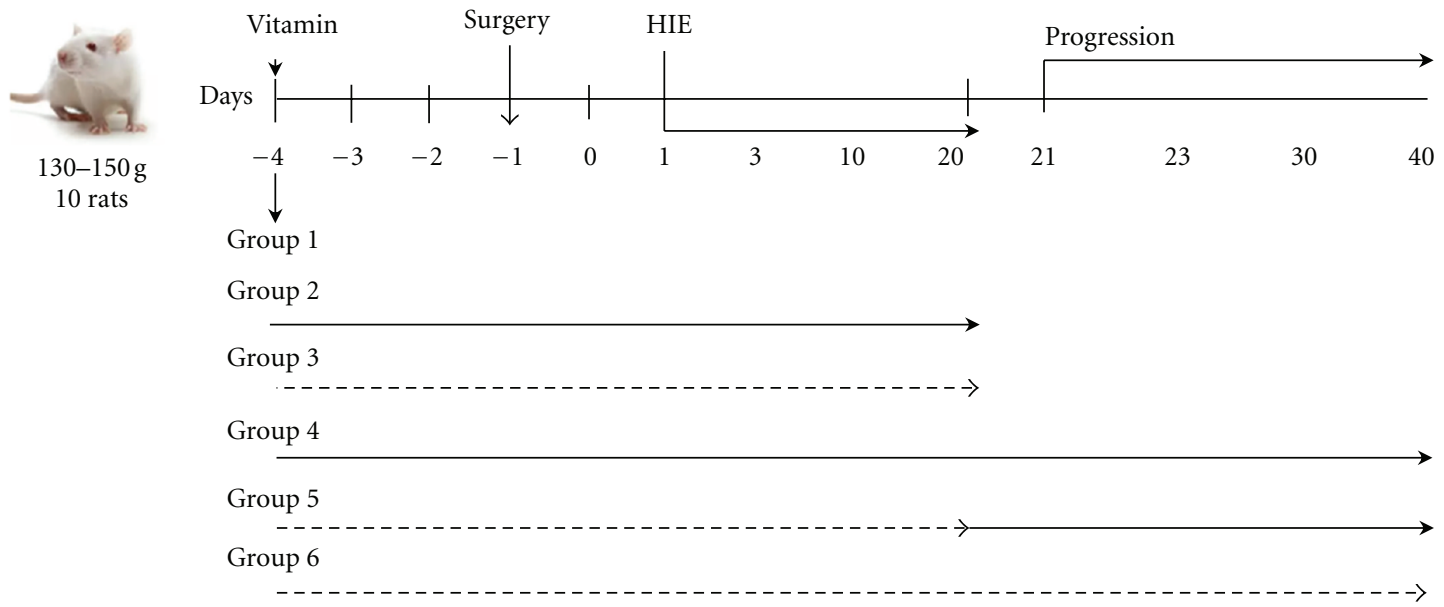

\footnotetext{
Group 1: Rats CTL

Group 2: OA rats with non vitamin supplementation (nV)

Group 3: OA rats supplemented with vitamin (sV)

Group 4: OA rats with no vitamin supplementation and progression with non vitamin supplementation $(\mathrm{nV}+\mathrm{PnV})$

Group 5: OA rats supplemented with vitamin and progression with non vitamin supplementation $(\mathrm{sV}+\mathrm{PnV})$

Group 6: OA rats supplemented with vitamin and progression supplemented with vitamin (sV + PsV)
}

FIGURE 1: Experimental groups. A schematic example of the 20-day induction group and the 40-day progression group of rats with OA with or without supplementation is shown. The same scheme was applied for the other induction ( 3 and 10 days) and progression (20 and 40 days) groups. Vitamin D (4 IU/kg/d) was administrated orally with an esophagogastric cannula 14 HALO. The arrow indicates the group without vitamin supplementation $(\mathrm{nV})$; the pointed arrow indicates the supplementation with vitamin $\mathrm{D}$ ( $\mathrm{sV}$ ). Each group was conformed by 10 rats, and every experiment was performed at least by triplicate.

Cartilage samples were obtained from 10 knees of healthy (control (CTL)) or OA rats per experimental group. The local ethics committee of the UPEAL, CINVESTAV-IPN (NOM-062-ZOO-1999), approved this study. The rats were anesthetized with a ketamine $60 \mathrm{mg} / \mathrm{kg}$ and xylazine $4 \mathrm{mg} / \mathrm{kg}$ solution, and OA was induced in the knee of the right hind leg by a partial meniscectomy, which is the transecting of the medial collateral ligament (MCL) [14], and high-impact exercise (HIE) for $1,3,6,8,10$, or 20 days. For the progression groups, the rats exercised for 3,10 , or 20 days and then were maintained for 20 additional days without exercise (Figure 1), resulting, study periods of 23,30 , and 40 days, respectively. Explants were obtained from the femoral condyles and tibial plateau cartilage from joints where the partial meniscectomy was performed.

The high-impact exercises began two days after surgery. The rats were forced to slide from side to side for 2 minutes in a box, and then the rats were dropped continuously for another 2 minutes. Finally, the rats were forced to jump as the box was continually shaken vertically for 1 minute. This exercise cycle was repeated 3 times each day.

2.2. Vitamin D Administration. Capsules of vitamin $\mathrm{D}(1,25-$ dihydroxyvitamin $\mathrm{D}_{3}$ ) from Member's Marck (Bentonville, $\mathrm{AR})$ were dissolved in corn oil (vehicle). The doses were $100 \mathrm{ng}$ ( $4 \mathrm{IU}), 1 \mu \mathrm{g}$ (40 IU), $10 \mu \mathrm{g}$ (400 IU), and $100 \mu \mathrm{g} / \mathrm{kg} /$ day (4000 IU) and were administrated orally with an esophagogastric cannula $14 \mathrm{~h}$ after lights on (14HALO [15]). Each dose was administered 3 days before surgery, and daily administration continued until the last day of $\mathrm{OA}$ induction. For the evaluation of $4 \mathrm{IU}$ of vitamin D supplementation during OA progression, three experimental subgroups were included: (1) rats without vitamin supplementation during $\mathrm{HIE}+$ progression without vitamin supplementation $(\mathrm{nV}+$ $\mathrm{PnV}$ ), (2) rats supplemented with the vitamin during HIE + progression without vitamin supplementation of vitamin $(\mathrm{sV}+\mathrm{PnV})$, and (3) rats supplemented with the vitamin during $\mathrm{HIE}+$ progression with vitamin supplementation $(\mathrm{sV}+$ PsV) (Figure 1).

2.3. RNA Isolation, cDNA Synthesis, and Real-Time Quantitative Polymerase Chain Reaction (PCR) Amplification. The cartilage samples were enzymatically digested, and the total RNA was isolated from chondrocytes for real-time reverse transcriptase PCR analysis (qPCR) of TLR-1, TLR-2, TLR-4, TLR-6, IL- $1 \beta$, TNF- $\alpha$, IL-6, MMP-3, MMP-9, and MMP-13 (Real-Time PCR System, model 7500, Applied Biosystems, Carlsbad, CA, USA) using the TRIzol LS Reagent (Invitrogen, USA). The first strand of cDNA was synthesized from $1.0 \mu \mathrm{g}$ total RNA using a high capacity cDNA reverse transcription kit with RNase inhibitor (Applied Biosystems, Carlsbad, CA, USA). Glyceraldehyde 3-phosphate dehydrogenase (GAPDH) mRNA was used as an endogenous control to allow for the relative quantification of the genes of interest. The qRT-PCR was performed with the fast SyBR-Green master mix (Applied Biosystems, Carlsbad, CA, USA) on both the targets and the endogenous control (the probes used are shown in Table 1). The amplified PCR products were 
TABLE 1: Probes used for genes expression.

\begin{tabular}{|c|c|c|}
\hline Gene & Sense & Antisense \\
\hline TLR1 & 5'-TACCCTGAACAACGTGGACA-3' & 5'-ATCGACAAAGCCCTCAGAGA-3' \\
\hline TLR2 & $5^{\prime}$-GGAGACTCTGGAAGCAGGTG-3' & 5'-CGCCTAAGAGCAGGATCAAC-3' \\
\hline TLR4 & 5'-CCAGAGCCGTTGGTGTATCT-3' & 5'-TCAAGGCTTTTCCATCCAAC-3' \\
\hline TLR6 & 5'-GTCTCCCCACTTCATCCAGA-3' & 5'-CCCACGTTTACССТTCTCAA-3' \\
\hline $\mathrm{TNF} \alpha$ & 5'-AAACTCGAGTGACAAGCCCG-3' & 5'-GCAGCCTTGTCCCTTGAAGA-3' \\
\hline $\operatorname{IL} 1 \beta$ & 5'-CACCTCTCAAGCAGAGCACAG-3' & 5'-GGGTTCCATGGTGAAGTCAAC-3' \\
\hline IL6 & 5'-TCCTACCCCAACTTCCAATGCTC-3' & 5'-TTGGATGGTCTTGGTCCTTAGCC-3' \\
\hline MMP-3 & 5'-AGACAGGCACTTTTGGCG-3' & 5'-CTTCATGACCTCGGATAGCC-3' \\
\hline MMP-9 & $5^{\prime}$-TCGAATCACGGAGGAAGC-3' & $5^{\prime}$-CCTAGCCССАACTTATCCAG-3' \\
\hline MMP-13 & $5^{\prime}$-CAAGGACCCTGGAGCCCTGA-3' & $5^{\prime}$-TGAGGGTGCAGACGCCAGA-3' \\
\hline GAPDH & $5^{\prime}$-TCCTACCCCCAATGTATCCG-3' & $5^{\prime}$-GGTGGAAGAATGGGAGTTGC-3' \\
\hline
\end{tabular}

quantified by measuring the calculated cycle thresholds $\left(\mathrm{C}_{\mathrm{T}}\right)$. The amounts of specific mRNA in the samples were calculated by the $\Delta \Delta \mathrm{C}_{\mathrm{T}}$ method. The mean value of the CTL chondrocytes target levels was used as the calibrator (one per sample), and the results were expressed as the $n$-fold difference relative to normal controls for the relative expression level data $\left(2^{-\Delta \Delta \mathrm{C}_{\mathrm{T}}}\right)$.

The quality of the samples was determined by the difference in the absorbance at 260 and $280 \mathrm{~nm}$. The RNA had an average A260/A280 ratio of 1.87. The RNA quality was also assessed by the electrophoresis of the total RNA followed by staining with ethidium bromide. In all samples, bands of $5 \mathrm{~kb}$ and $2 \mathrm{~kb}$, corresponding to the $28 \mathrm{~S}$ and $18 \mathrm{~S}$ rRNAs, were observed. The cDNA obtained had an A260/A280 average value of 1.86 .

2.4. Histological Analysis. Cartilage samples were obtained from 2 knees of healthy (control; CTL) or 20 days OA rats with or without vitamin D supplementation. Samples from each group were fixed with $4 \%$ formol, embedded in paraffin, cut at a thickness of $4 \mu \mathrm{m}$ and stained with hematoxylin and eosin (HE).

2.5. Statistical Analysis. The data are shown as the mean \pm the standard error (standard error of the mean; SEM). The statistical significance is indicated on the graphs as follows: $P<0.05\left(^{*}\right), P<0.01\left(^{* *}\right)$, or $P<0.001\left(^{* * *}\right)$, with a $95 \%$ CI. The expression levels of TLRs, MMPs, and cytokines were measured in five independent experiments $(n=5)$ for the OA kinetic analysis and in three independent experiments $(n=3)$ for the vitamin $\mathrm{D}$ dose-response and the OA progression analysis. The statistical analyses were performed by the Graph Pad Instat program (Graph Pad Software Inc., San Diego, CA, USA). A two-way analysis of variance (ANOVA) followed by Tukey's post hoc test was performed to compare means between the experimental groups without vitamin supplementation and the CTL group or a test for linear trend when applicable. A one-way ANOVA analysis using Dunnet's post hoc test was performed to compare the different doses of vitamin D. Student's $t$-test was used to compare between the progression groups without vitamin supplementation and the progression group supplemented with vitamin D since the induction. Finally, the correlation analysis was performed using Pearson's correlation.

\section{Results}

3.1. Expression of TLRs, Proinflammatory Cytokines, and $M M P$ Genes in Chondrocytes during OA Induction. To evaluate TLR-1, TLR-2, TLR-4, and TLR-6 gene expression during OA development in this rat model, we isolated cartilage chondrocytes from rats subjected to $1,3,6,8,10$, and 20 days of OA induction and assessed TLR mRNA levels using quantitative RT-PCR. Figure 2(a) shows that TLR-1, TLR-2, and TLR- 6 were expressed in the chondrocytes throughout the $\mathrm{OA}$ induction with no differences in expression levels compared to the control group. Meanwhile, an increase in the level of TLR-4 mRNA expression was observed (linear trend, $\left.P=0.0041 ; R^{2}=0.8339\right)$ from day $8(1.6$-fold; $P<0.05)$ to 20 (2.1-fold; $P<0.001)$ with maximal expression on day 10 (2.8-fold; $P<0.001)$.

TLR-4 activation results in the expression of molecules associated with damage, such as MMPs and inflammatory cytokines. Therefore, we evaluated the mRNA expression of MMP-3, MMP-9, MMP-13, IL-6, TNF- $\alpha$, and IL-1 $\beta$. The expression of MMP-9 and MMP-13 was detected during the progression of $\mathrm{OA}$, but there were no major changes in their expression between the OA and the CTL groups (Figure 2(b)). In contrast, MMP- 3 expression was higher in the OA groups on days 8 (1.8-fold; $P<0.01)$ and 10 (2.1-fold; $P<0.01$ ), with a linear trend suggesting an increase in expression $\left(R^{2}=\right.$ 0.5883; $P=0.0219$ ), as shown in Figure 2(b). Similar to MMP-9 and MMP-13 expression, the IL- 6 expression level remained similar to that of CTL, while the expression level of TNF- $\alpha\left(R^{2}=0.4173\right)$ and IL- $1 \beta\left(R^{2}=0.375\right)$ was elevated throughout OA induction (Figure 2(c)). There was a peak in the IL- $1 \beta$ expression on day 8 (18-fold; $P<0.001$ ), suggesting that this cytokine plays an important role during the onset of OA pathogenesis.

TLR-4 expression correlated with the expression of MMP-3 $\left(R^{2}=0.7692 ; P=0.0095\right)$, TNF- $\alpha\left(R^{2}=0.7127\right.$; $P=0.0169)$, and IL-1 $\beta\left(R^{2}=0.3954 ; P=0.1304\right)$, as shown in Supplementary Figure 1, (see Supplementary Material available online at doi:10.1155/2012/156563). These results 


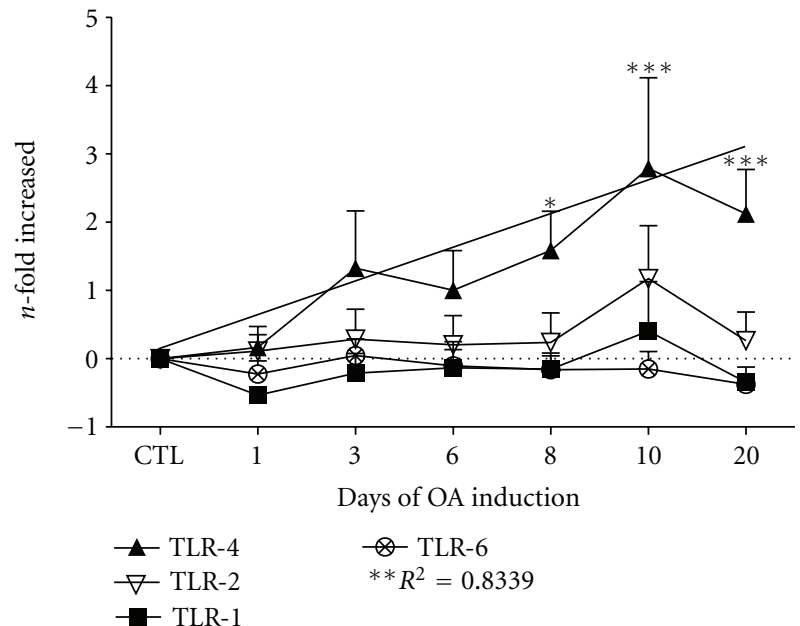

(a)

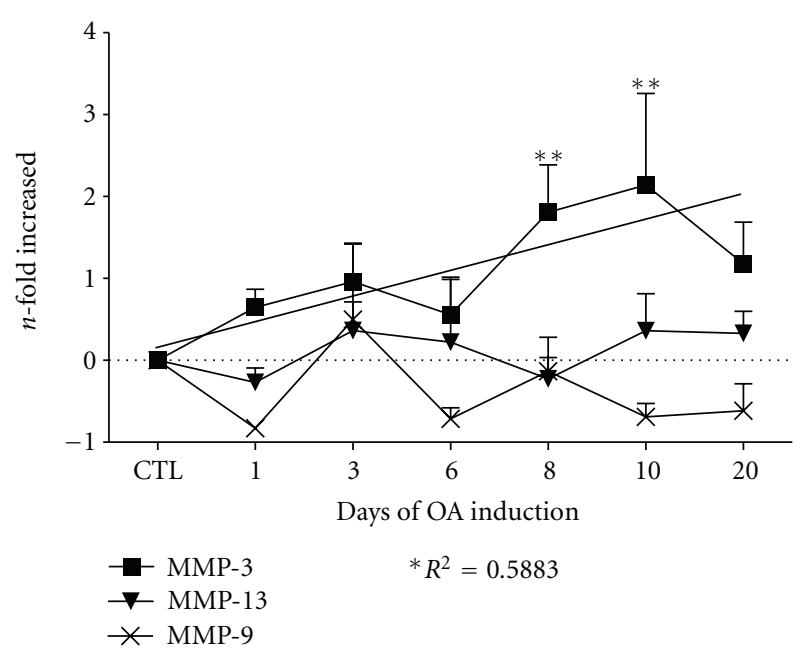

(b)

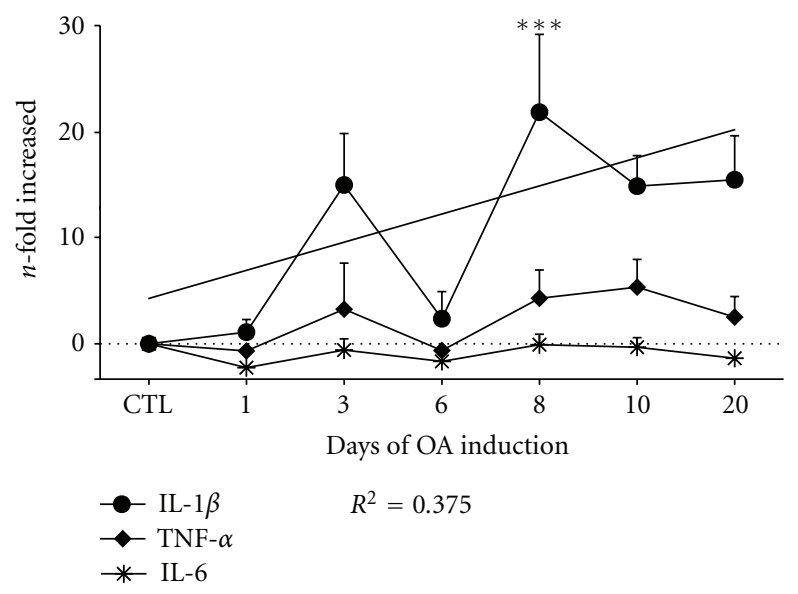

(c)

Figure 2: TLR-4, MMP-3, IL-1 $\beta$, and TNF- $\alpha$ expression was induced during osteoarthritis induction. Chondrocytes were collected and assayed for gene expression by real-time RT-PCR. The relative expression levels were normalized to the expression of the housekeeping GAPDH gene expression and presented as the $n$-fold difference. (a) TLR-2 and TLR-1 showed a similar pattern of expression during OA induction, whereas TLR- 6 was only expressed during the acute stage of OA. TLR- 4 showed a remarkable increase in expression with respect to the CTL group throughout OA induction, with a significant linear trend $\left(R^{2}=0.8339 ; P=0.0041\right)$. (b) The relative expression levels of MMP-3, MMP-9, and MMP-13, normalized to GAPDH gene expression, are shown. MMP-3 expression increased during OA induction $\left(R^{2}=0.5883, P=0.0219\right)$ and was highest on days 8 ( 1.8 -fold; $95 \%$ CI 0.005241 to 3.609 ) and 10 (2.1-fold; $95 \%$ CI 0.3375 to 3.941$)$. (c) The relative expression of IL- $1 \beta$, IL- 6 , and TNF- $\alpha$, normalized to GAPDH gene expression, is shown. IL- $1 \beta$ gene expression increased during OA induction $\left(R^{2}=0.375\right)$, while TNF- $\alpha$ gene expression changed modestly $\left(R^{2}=0.4173\right)$, with the highest increase on day 10 (1.6fold). The results are pooled data from five independent experiments with 50 total rats. The asterisks indicate ${ }^{*} P<0.05$, ${ }^{* *} P<0.01$, and ${ }^{* * *} P<0.001$, which were determined using a 2-way ANOVA followed by Tukey's post hoc test to compare the experimental groups with the CTL group. The error bars indicate the SEM.

suggest that the activation of the TLR- 4 pathway plays an important role in OA pathogenesis by inducing MMP-3, TNF- $\alpha$, and IL- $1 \beta$ expression. However, IL- $1 \beta$ expression may not be entirely dependent on TLR- 4 because it also depends on the NOD-like receptors pathway [16].

3.2. Effect of Vitamin D on OA Cartilage (Dose Response). If the activation of TLRs and the synthesis of cytokines and MMPs are associated with OA and if vitamin D modulates TLR- 4 activation, then there should be detectable changes in the joint in response to such modulation. Some of the main characteristics of OA are joint hypertrophy and cartilage and bone erosion, which are associated with increased fibrotic tissue at the borders of the joint. For that reason, we next evaluated the effect of supplementation with different vita$\min \mathrm{D}$ doses $(4,40,400$, and $4000 \mathrm{IU} / \mathrm{kg} / \mathrm{d})$ during the induction of OA for 20 days (OA20). We measured the OA condyles and nonmeniscected condyles (right and left, resp.), and the difference between them was considered an indicative value of OA hypertrophy severity (Figures $3(\mathrm{a})$ and $3(\mathrm{~b})$ ). The wideness of the condyles in OA20 rats without vitamin D 


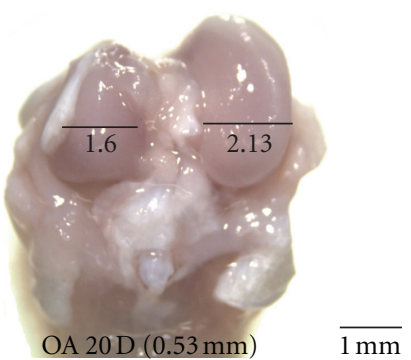

(a)

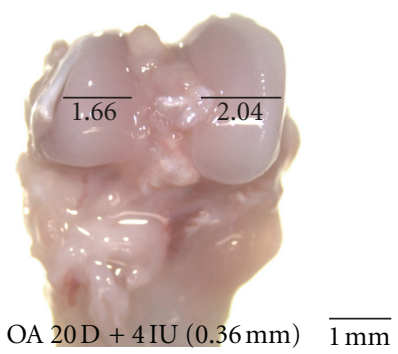

(b)

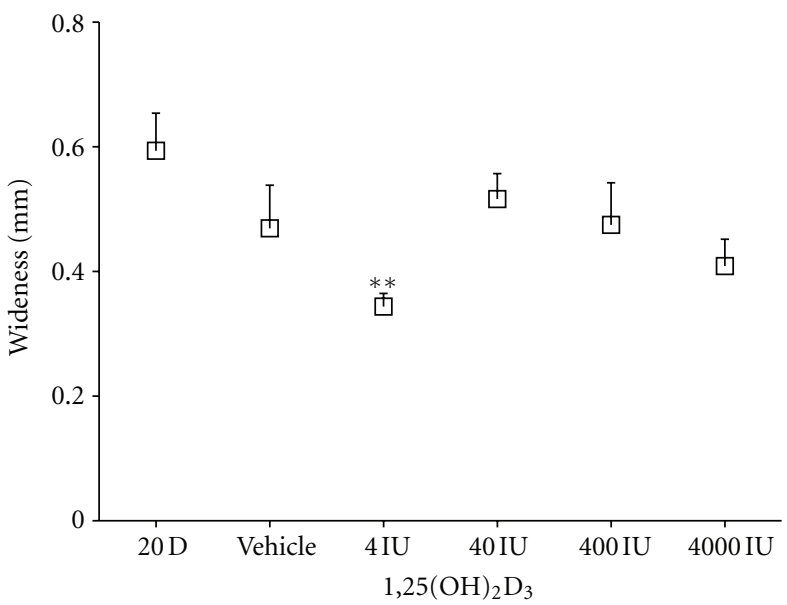

(c)

Figure 3: Vitamin D reduces the wideness of OA condyles. (a) A representative image of the femoral condyles from a rat with 20 days of OA without treatment. (b) A representative image of the femoral condyles from a rat treated with $4 \mathrm{IU} / \mathrm{kg} /$ day of vitamin D. The difference in wideness was taken as an indicator of the degree of severity of OA hypertrophy. (c) The graph presents the average wideness at a given dose from 3 different experiments with 15 total rats. A decrease in the severity of $0.34 \pm 0.047 \mathrm{~mm}(95 \%$ CI 0.05367 to 0.4459 , $* * P<0.01)$ was determined using a one-way ANOVA with Dunnet's post hoc test to compare all of the conditions with the control group (OA of 20 days without vitamin D). The error bars indicate the SEM. The scale bar $=1 \mathrm{~mm} 8 \mathrm{x}$.

was used as the control (Figure 3(a)). We observed that the $4 \mathrm{IU} / \mathrm{kg} /$ day dose was the only dose that caused a reduction in the wideness of the OA condyles $(P<0.01 ; 0.34 \pm 0.047 \mathrm{~mm})$ (Figure 3(c)).

\subsection{Effect of Vitamin D Supplementation on OA Induction.} To assess whether the protective effect of supplementation with 4 IU of vitamin D involves TLR- 4 modulation, we first evaluated the expression of TLR4, IL- $1 \beta$, TNF- $\alpha$, and MMP3 during different stages of $\mathrm{OA}$ induction. We established day 3 of induction as the acute stage, day 10 as the intermediate stage, and day 20 as the chronic stage. This classification was established taking in consideration the previous results [14]. TLR-4 expression was upregulated (5.89-fold; $P=0.0102$ $F$ test) until the chronic stage (Figure 4(a)), while MMP3 expression was upregulated from the intermediate stage (4.79-fold; $P=0.0246 F$ test) to the chronic stage (4.13-fold; $P=0.0116 F$ test, Figure $4(\mathrm{~b}))$. TNF- $\alpha$ was also upregulated during the intermediate and chronic stages (10 days: $12.75-$ fold; $P=0.0004$ and 20 days: 5.31 -fold; $P=0.0041 F$ test), as shown in Figure $4(\mathrm{c})$. Meanwhile, IL- $1 \beta$ expression was downregulated in the acute stage (2.21-fold; $P=0.0104 F$ test), which was followed by an upregulation on day 10 (12.75-fold; $P=0.0473 F$ test). Finally, IL- $1 \beta$ showed the same levels of expression in the chronic stage (5.31-fold; $P=0.2422 F$ test) as the $\mathrm{nV}$ group (Figure $4(\mathrm{~d})$ ). These data suggested that the protective effect $(P=0.0010 t$ test $)$ of vitamin $\mathrm{D}$ supplementation observed during $\mathrm{OA}$ induction (Figures 4(e) and 4(f) and Supplementary Figure 2) is not due to the modulation of TLR- 4 activation at the transcriptional level. In Figure 4(f), we can observe after 20 days of OA induction a severe damage with intense hypertrophy and hyperplasia of the cartilage, which diminished with vitamin D supplementation.

3.4. Effect of Vitamin D Supplementation on Gene Expression during $O A$ Progression. To mimic the natural progression of $\mathrm{OA}$, we evaluated the effect of vitamin $\mathrm{D}$ supplementation in rats with OA progression, as described in Figure 1. The expression of TLR-4 was downregulated at day 30 (28.61fold; $P=0.0125 F$ test) in the $\mathrm{sV}+\mathrm{PsV}$ group compared to the $n V+P n V$ group (Figure 5(a)). MMP-3 expression was similar between the evaluated groups (Figure 5(b)); while, TNF- $\alpha$ expression was downregulated in the $s \mathrm{~V}+\mathrm{PsV}$ group on day 30 compared to the groups: $s \mathrm{~V}+\mathrm{PnV}$ (39.55-fold; $P=0.0042 F$ test) and $n V+\operatorname{PnV}(3.66$-fold; $P=0.0244 F$ test) (Figure $5(\mathrm{c}))$. In addition, IL- $1 \beta$ was downregulated on day 40 (51.97-fold; $P=0.0168 F$ test) in the $s V+P s V$ group compared to the $\mathrm{sV}+\mathrm{PnV}$ group (Figure 5(d)) and upregulated on day 23 (1.09-fold; $P=0.0491 F$ test) compared to the $\mathrm{nV}+\mathrm{PnV}$ group (Figure 5(d)).

In contrast to the protective effect of vitamin $\mathrm{D}$ observed during the $\mathrm{OA}$ induction, we did not find any evidence for a protective effect of vitamin $\mathrm{D}$ supplementation during OA progression. As is shown in Figure 5(e) and Supplementary Figure 2, the rats of all groups had the same degree of damage from $\mathrm{OA}$ at the end of the experiment. In this case, the damage was correlated with the changes observed in the gene expression of TLR-4, MMP-3, and TNF- $\alpha$ (Figure 5). 


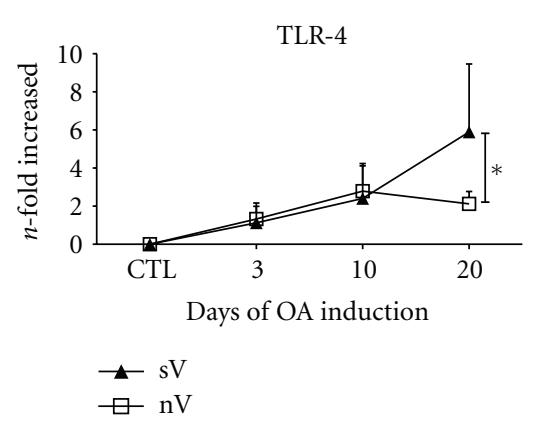

(a)

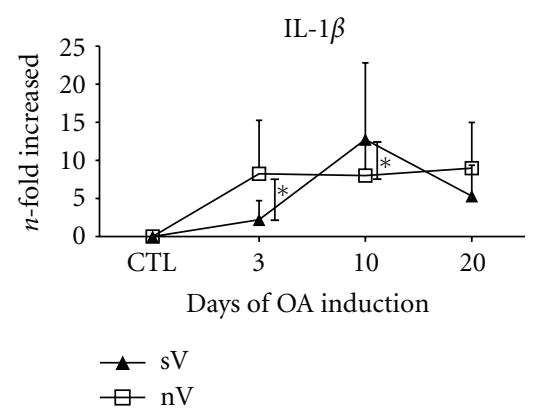

(d)

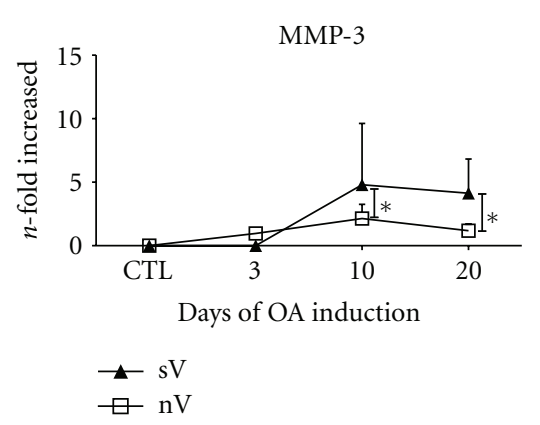

(b)

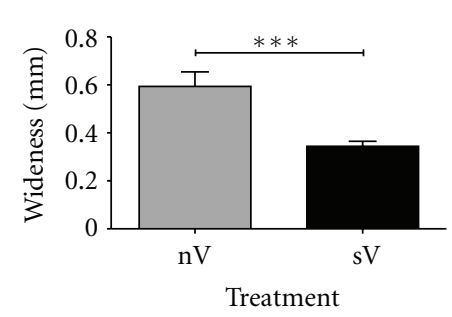

(e)

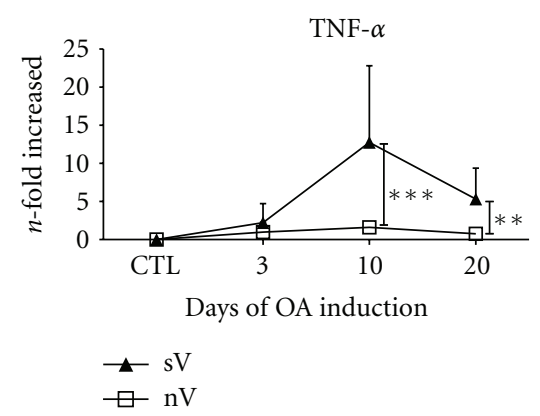

(c)
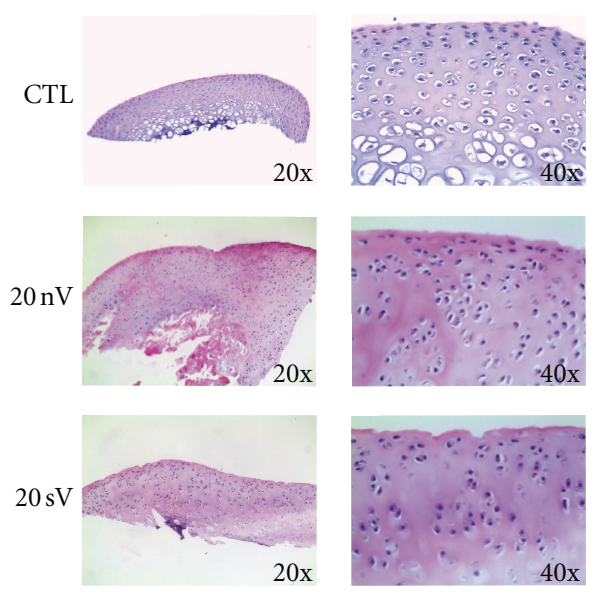

(f)

FIGURE 4: The protective effect of vitamin D is independent of the transcriptional modulation of TLR-4. The evaluation was performed during the OA induction period for (a)-(d). The gene expression of TLR4 (a), MMP-3 (b), TNF- $\alpha$ (c), and IL- $1 \beta$ (d) from rats in the nV group $(\square)$ compared with the sV group $(\boldsymbol{\Lambda})$ is shown. The gene expression levels were normalized to GAPDH gene expression and presented as the $n$-fold difference in expression. (e) A comparison of the hypertrophy (wideness) of the condyles from rats with 20 days of OA that were supplemented with vitamin $\mathrm{D}(\mathrm{sV})$ to that of rats with no supplementation $(\mathrm{nV})$. (f) A morphological comparison between condyles of control rats and rats with 20 days of OA induction treated with vitamin $\mathrm{D}(\mathrm{sV})$ and nontreated (nV) by HE stain. The supplementation was performed daily with $4 \mathrm{IU}$ of vitamin $\mathrm{D}$. The results are expressed as pooled data from three independent experiments with 16 to 18 total rats $(\mathrm{a}-\mathrm{d})$ or with 6 rats per group (e). The error bars indicate the SEM. A $t$-test with an $F$ test was performed to obtain the indicated statistical significance values: ${ }^{*} P<0.05,{ }^{* *} P<0.01$, and ${ }^{* * *} P<0.001$. Abbreviations indicate the following treatments: $\mathrm{nV}$ (without vitamin supplementation); $\mathrm{sV}$ (with vitamin supplementation).

\section{Discussion}

The present study shows the involvement of the TLR-4 pathway in the expression of MMP-3, IL- $1 \beta$, and TNF- $\alpha$ during the development of OA. It also shows that vitamin $\mathrm{D}$ supplementation has a protective effect on condyle wideness during the induction of OA despite an increase in the expression of the above genes. In contrast, during OA progression, vitamin $\mathrm{D}$ supplementation induced the downregulation of TLR- 4 , IL- $1 \beta$, and TNF- $\alpha$ on day 30 , but this had no beneficial effect on the disease outcome.

Although human articular chondrocytes express mRNAs for all TLRs (TLRs 1-9) [17] only TLR-2 and TLR-4 expression increased in OA chondrocytes. OA chondrocytes stimulated in vitro with ligands for TLR- 2 or IL- $1 \beta$ induced the release of $\mathrm{PGE}_{2}, \operatorname{MMPs}(-1,-3$, and -13$)$, and nitric oxide as a consequence of this stimulation [18-20]. Similarly, the stimulation of TLR-4 expression in normal chondrocytes $[17,21]$, induces the secretion of TNF- $\alpha$, IL- $1 \beta$, MMP-13, and inducible nitric oxide synthase [22]. In this study, we found that TLR-4 was upregulated during OA induction in vivo and that its expression correlates with TNF- $\alpha$, IL- $1 \beta$, and MMP-3 gene expression. These data suggest that TLR4 signaling is important in the pathophysiological response that causes the inflammation and degradation of the cartilage in OA.

The TLR activation pathway induces the expression of the vitamin D receptor (VDR) and CYP27B1 ( $1 \alpha$-hydroxylase), the enzyme that converts 25-hydroxycholecalciferol into $1,25(\mathrm{OH})_{2} \mathrm{D}_{3}$, thereby promoting innate immunity through VDR activation $[23,24]$. It has been reported that p65, a subunit of NF- $\kappa \mathrm{B}$, interacts directly with the VDR and that this interaction modulates the VDR and TLR responses [25-27]. The outcome of this TLR-VDR transcriptional partnership is the regulation of the target genes of both receptors. For this reason, we decided to evaluate if the suggested protective effect of vitamin $\mathrm{D}$ supplementation on OA was due to a modulation of TLRs, cytokines, and MMPs at 


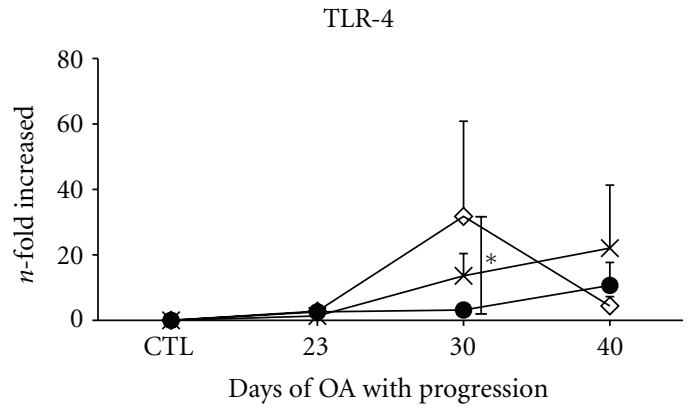

(a)

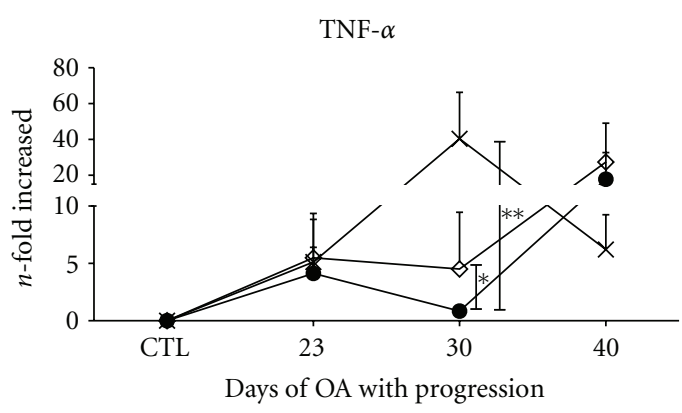

(c)

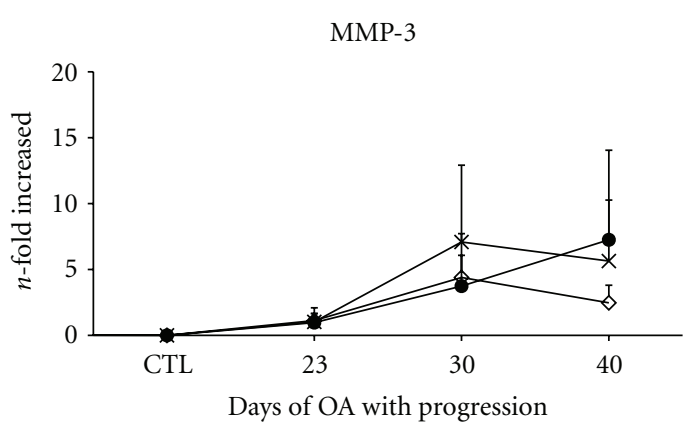

(b)

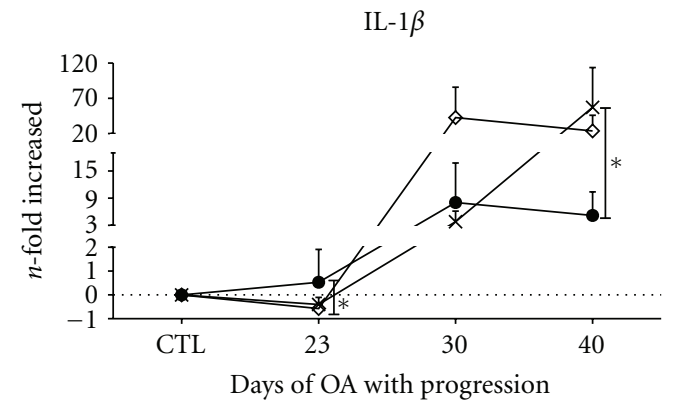

(d)

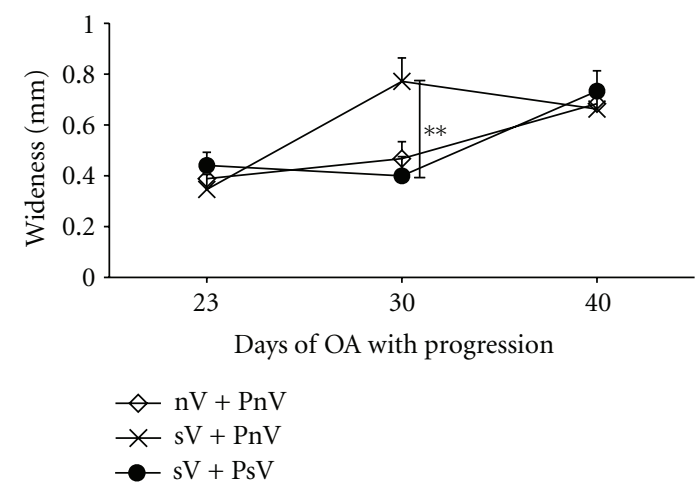

(e)

Figure 5: Vitamin D has no effect on OA severity. The evaluation of OA was performed during the OA progression phase.(a)-(d). The gene expression of TLR-4 (a), MMP-3 (b), TNF- $\alpha$ (c), and IL-1 $\beta$ (d) from rats in the $\mathrm{sV}+$ Psv group $(\bullet)$ compared with the sV + PnV $(\times)$ and the $\mathrm{nV}+\operatorname{PnV}(\diamond)$ groups. The gene expression levels were normalized to GAPDH gene expression and are presented as the $n$-fold difference in expression. (e) A comparison of the hypertrophy (wideness) of condyles from the rats of the sV $+\mathrm{PsV}(\bullet), \mathrm{sV}+\mathrm{PnV}(\times)$ and $\mathrm{nV}+\mathrm{PnV}(\diamond)$ groups is shown. The results shown are pooled data from three independent experiments with 16 to 18 total rats $(\mathrm{a}-\mathrm{d})$ or with 12 to 16 total rats (e). The error bars indicate the SEM. A $t$-test with an $F$ test was performed; the notations indicate $* P<0.05$ and $* * P<$ 0.01 . The following groups were included: $\mathrm{SV}+\mathrm{PsV}$ (vitamin supplementation during induction and progression with supplementation of vitamin); $\mathrm{sV}+\mathrm{PnV}$ (vitamin supplementation during induction and progression without vitamin supplementation); $\mathrm{nV}+\mathrm{PnV}$ (without vitamin supplementation during induction and progression without vitamin supplementation). For the supplement, 4 IU of vitamin D was administrated daily.

the transcriptional level [1] or if vitamin D was in fact associated with more severe OA because VDR expression has also been associated with MMP-3 expression in OA cartilage [28] and the upregulation of inflammatory cytokines [29]. In this study, we observed that vitamin $\mathrm{D}$ supplementation differentially modulated the gene expression of TLR-4, TNF- $\alpha$, IL- $1 \beta$, and MMP-3 during OA induction and progression. Vitamin D supplementation induced TLR-4 expression during $\mathrm{OA}$ induction but downregulated it during
OA progression. These antagonistic responses might be due to the continued mechanical overload that promotes the activation of the inflammatory pathway during OA induction.

This differential response has been observed after inflammatory cytokines modulation. Vitamin D downregulates IL- $1 \beta$ and TNF- $\alpha$ expression in peripheral blood mononuclear cells via TLR-4 downregulation [22]. In contrast, in human monocyte-derived macrophages activated with 
lipopolysaccharide, vitamin $\mathrm{D}$ stimulates the production of IL-1 $\beta[28]$ and downregulates TNF- $\alpha$ expression $[1,22,30]$. In our results, we observed both positive and negative effects on cytokines. IL-1 $\beta$ increased on day 10 of OA induction $(\mathrm{sV})$ and was downregulated on day 40 of OA progression $(\mathrm{sV}+\mathrm{PsV})$. Similar effects on TNF- $\alpha$ expression were observed.

Enhancing and suppressive roles for vitamin D have also been described for MMPs expression. Vitamin D has been associated with MMP-3 expression in human OA cartilage. In vitro studies show that vitamin $\mathrm{D}$ induces the production of MMP-1, MMP-3, and $\mathrm{PGE}_{2}$ in chondrocytes stimulated with IL-1 $\beta$, whereas it reduces MMP-1, MMP-3, and $\mathrm{PGE}_{2}$ induction by IL- $1 \beta$ in rheumatoid synovial fibroblasts $[28,31]$, which are two scenarios that could be present and contribute to the final state of the cartilage during OA development. Our in vivo results agree with the effect described for MMP-3 in chondrocytes in vitro with regard to the groups of OA induction and at day 40 of OA progression. However, despite that at day 40, MMP-3 expression was upregulated in OA rats, there were no major changes in the other groups. These data suggest that the effect of vitamin D depends not only on the cell type but also on the activation state of the cells and the inflammatory environment.

The regulatory modulation leads to the hypertrophy of the articular cartilage, which is characterized by physical changes, such as fraying, fibrillations with a rough surface, and the formation of osteophytes that become more prominent as the disease progresses [28]. We evaluated hypertrophy in our model by comparing the width of the right and left condyles and observed that the condyle wideness (in millimeters) was not different except when comparing the 30-day group treated with vitamin $\mathrm{D}$ during OA progression $(\mathrm{sV}+\mathrm{PsV})$ to the 30-day group supplemented only during the induction $(\mathrm{sV}+\mathrm{PnV})$. The comparison of these groups also revealed that vitamin $\mathrm{D}$ leads to the downregulation of TLR-4, MMP-3, and TNF- $\alpha$. These findings are in accordance with a study indicating that vitamin D deficiency is greatly associated with the early stages of OA but absent in the advanced stages [4]. This could be explained by a recent report indicating that vitamin $\mathrm{D}$ precedes the TLR activation, with no effect observed when vitamin $D$ is administrated at the same time or after TLR stimulation [32]. These data and our data suggest that vitamin $\mathrm{D}$ supplementation must be administrated before cartilage damage occurs.

Remarkably, we observed hypertrophy protection with low doses of vitamin D (4 IU) that was not observed in the same extends with higher doses (40-4000 IU). This lack of dose response might be due to the dual effect that vitamin D has on the different cells of the joint $[28,31]$. In fact, it has been reported that administration of high doses of vitamin $\mathrm{D}$ induces side effects like hypercalcemia and hyperphosphatemia [15]. However, there is not an appropriate pharmacokinetic study that establishes the effects of vitamin D on the cartilage. Additionally, there are other factors that have to be taken into consideration, including the vitamin $\mathrm{D}$-dependent induction of the expression of VEGF, growth factors [33], and MMPs $[31,33,34]$. These findings suggest that, in addition to its regulation of inflammation and damage, vitamin
D could also contribute to factors highly associated with OA progression $[28,35]$.

In summary, our findings suggest that vitamin D may play a key role in the pathophysiology of OA. Additionally, the effects of vitamin D on OA may reflect the state of cell activation. Further studies are needed to examine the effects of vitamin D on the various molecules associated with the key degenerative or reparative processes within osteoarthritic cartilage. In addition, it is important to establish whether vitamin D supplementation in healthy subjects with serologically adequate levels of vitamin $\mathrm{D}$ yields a better response than supplementation in subjects with lower serum levels. However, it has been reported that healthy women with higher levels of vitamin $\mathrm{D}$ in serum had lower concentrations of TNF- $\alpha$ [36]. Moreover, a pharmacokinetic would help to understand the kinetics and effects of different doses of vitamin D.

\section{Abbreviations}

\begin{tabular}{|c|c|}
\hline OA: & Osteoarthritis \\
\hline HIE: & High-impact exercise \\
\hline TLRs: & Toll-like receptors \\
\hline CTL: & Control \\
\hline IL- $1 \beta$ : & Interleukin- $1 \beta$ \\
\hline IL-6: & Interleukin-6 \\
\hline TNF $\alpha$ : & Tumor necrosis factor alpha \\
\hline MMP: & Matrix metalloproteinase \\
\hline DAMPs: & Damage-associated molecular patterns \\
\hline IU: & International units \\
\hline $1,25(\mathrm{OH})_{2} \mathrm{D}_{3}:$ & Vitamin D \\
\hline 14 HALO: & 14 hours after light on \\
\hline qPCR: & $\begin{array}{l}\text { Quantitative real-time polymerase chain } \\
\text { reaction }\end{array}$ \\
\hline HE: & Hematoxylin and eosin \\
\hline sV: & Rats supplemented with the vitamin \\
\hline $\mathrm{nV}:$ & Rats without vitamin supplementation \\
\hline $\mathrm{nV}+\mathrm{PnV}:$ & $\begin{array}{l}\text { Rats without vitamin supplementation } \\
\text { during HIE + progression without vitamin } \\
\text { supplementation }\end{array}$ \\
\hline$s \mathrm{~V}+\mathrm{PnV}:$ & $\begin{array}{l}\text { Rats supplemented with the vitamin during } \\
\text { HIE + progression without vitamin } \\
\text { supplementation }\end{array}$ \\
\hline$s V+P s V:$ & $\begin{array}{l}\text { Rats supplemented with the vitamin during } \\
\text { HIE + progression with vitamin } \\
\text { supplementation }\end{array}$ \\
\hline ANOVA: & Analysis of variance \\
\hline GAPDH: & Glyceraldehyde 3-phosphate dehydrogenase \\
\hline $\mathrm{C}_{\mathrm{T}}:$ & Cycle threshold \\
\hline SEM: & Standard error of the mean \\
\hline NOD: & $\begin{array}{l}\text { Nucleotide oligomerization domain-like } \\
\text { receptors. }\end{array}$ \\
\hline
\end{tabular}

\section{Authors' Contribution}

E. C. Castillo conducted all the experiments, analyzed the data, conceived and designed the study, confirmed the data analysis, and wrote the initial draft of the manuscript. J. B. Kouri and V. Ortiz-Navarrete conceived of and designed 
study, confirmed the data analysis and reviewed the paper critically for important intellectual content. M. A. Hernandez-Cueto conducted the histopathological analysis. M. A. Vega-Lopez and C. Lavalle reviewed the paper critically for important intellectual content. All the authors read and approved the final paper.

\section{Conflict of Interests}

All authors declare that they have no competing interests.

\section{Acknowledgments}

The authors thank the support of the CONACyT and CINVESTAV-IPN, Zacatenco. They thank Daniel Hernandez Cueto for his technical assistance. This work was supported by CONACyT Grant 59787 (to J. B. Kouri). E. C. Castillo was supported by a CONACyT scholarship 192043 and a CINVESTAV scholarship.

\section{References}

[1] K. Sadeghi, B. Wessner, U. Laggner et al., "Vitamin D3 downregulates monocyte TLR expression and triggers hyporesponsiveness to pathogen-associated molecular patterns," European Journal of Immunology, vol. 36, no. 2, pp. 361-370, 2006.

[2] M. Haroon, U. Bond, N. Quillinan, M. J. Phelan, and M. J. Regan, "The prevalence of vitamin D deficiency in consecutive new patients seen over a 6-month period in general rheumatology clinics," Clinical Rheumatology, vol. 30, no. 6, pp. 789794, 2011.

[3] H. A. Bischoff-Ferrari, Y. Zhang, D. P. Kiel, and D. T. Felson, "Positive association between serum 25-hydroxyvitamin D level and bone density in osteoarthritis," Arthritis \& Rheumatism, vol. 53, no. 6, pp. 821-826, 2005.

[4] B. Heidari, P. Heidari, and K. Hajian-Tilaki, "Association between serum vitamin D deficiency and knee osteoarthritis," International Orthopaedics, vol. 35, no. 11, pp. 1627-1631, 2011.

[5] R. K. Chaganti, N. Parimi, P. Cawthon, T. L. Dam, M. C. Nevitt, and N. E. Lane, "Association of 25-hydroxyvitamin D with prevalent osteoarthritis of the hip in elderly men: the osteoporotic fractures in men study," Arthritis and Rheumatism, vol. 62, no. 2, pp. 511-514, 2010.

[6] T. E. McAlindon, D. T. Felson, Y. Zhang et al., "Relation of dietary intake and serum levels of vitamin $\mathrm{D}$ to progression of osteoarthritis of the knee among participants in the framingham study," Annals of Internal Medicine, vol. 125, no. 5, pp. 353-359, 1996.

[7] C. Ding, F. Cicuttini, V. Parameswaran, J. Burgess, S. Quinn, and G. Jones, "Serum levels of vitamin D, sunlight exposure, and knee cartilage loss in older adults: the Tasmanian older adult cohort study," Arthritis and Rheumatism, vol. 60, no. 5, pp. 1381-1389, 2009.

[8] D. T. Felson, J. Niu, M. Clancy et al., "Low levels of vitamin $\mathrm{D}$ and worsening of knee osteoarthritis: results of two longitudinal studies," Arthritis and Rheumatism, vol. 56, no. 1, pp. 129-136, 2007.

[9] R. T. Chlebowski, K. C. Johnson, D. Lane et al., "25-Hydroxyvitamin D concentration, vitamin D intake and joint symptoms in postmenopausal women," Maturitas, vol. 68, no. 1, pp. 73-78, 2011.
[10] S. Muraki, E. Dennison, K. Jameson et al., "Association of vitamin D status with knee pain and radiographic knee osteoarthritis," Osteoarthritis and Cartilage, vol. 19, no. 11, pp. 1301-1306, 2011.

[11] D. Jefferies, C. Farquharson, J. Thomson et al., "Differences in metabolic parameters and gene expression related to osteochondrosis/osteoarthrosis in pigs fed 25-hydroxyvitamin D3," Veterinary Research, vol. 33, no. 4, pp. 383-396, 2002.

[12] N. E. Lane, L. R. Gore, S. R. Cummings et al., "Serum vitamin $\mathrm{D}$ levels and incident changes of radiographic hip osteoarthritis: a longitudinal study. Study of osteoporotic fractures research group," Arthritis Rheum, vol. 42, no. 5, pp. 854-860, 1999.

[13] P. J. DeMarco, F. Constantinescu, L. D. Carbone, K. D. Barrow, and M. C. Nevitt, "Does vitamin D supplementation contribute to the modulation of osteoarthritis by bisphosphonates? Comment on the article by Carbone et al," Arthritis and Rheumatism, vol. 52, no. 5, pp. 1622-1623, 2005.

[14] K. Abbud and J. Kouri, "A novel rat osteoarthrosis model to assess apoptosis and matrix degradation," Pathology Research and Practice, vol. 196, no. 11, pp. 729-745, 2000.

[15] S. Tsuruoka, K. Nishiki, K. Sugimoto, and A. Fujimura, "Time of day improves efficacy and reduces adverse reactions of vitamin D3 in 5/6 nephrectomized rat," Life Sciences, vol. 71, no. 15, pp. 1809-1820, 2002.

[16] K. H. G. Mills and A. Dunne, "Immune modulation: IL-1, master mediator or initiator of inflammation," Nature Medicine, vol. 15, no. 12, pp. 1363-1364, 2009.

[17] K. Bobacz, I. G. Sunk, J. G. Hofstaetter et al., "Toll-like receptors and chondrocytes: the lipopolysaccharide-induced decrease in cartilage matrix synthesis is dependent on the presence of toll-like receptor 4 and antagonized by bone morphogenetic protein 7," Arthritis and Rheumatism, vol. 56, no. 6, pp. 1880-1893, 2007.

[18] S. L. Su, C. D. Tsai, C. H. Lee, D. M. Salter, and H. S. Lee, "Expression and regulation of Toll-like receptor 2 by IL- $1 \beta$ and fibronectin fragments in human articular chondrocytes," Osteoarthritis and Cartilage, vol. 13, no. 10, pp. 879-886, 2005.

[19] H. A. Kim, M. L. Cho, H. Y. Choi et al., "The catabolic pathway mediated by Toll-like receptors in human osteoarthritic chondrocytes," Arthritis and Rheumatism, vol. 54, no. 7, pp. 21522163, 2006.

[20] R. Liu-Bryan, K. Pritzker, G. S. Firestein, and R. Terkeltaub, "TLR2 signaling in chondrocytes drives calcium pyrophosphate dihydrate and monosodium urate crystal-induced nitric oxide generation," Journal of Immunology, vol. 174, no. 8, pp. 5016-5023, 2005.

[21] G. M. Campo, A. Avenoso, S. Campo, A. D’Ascola, G. Nastasi, and A. Calatroni, "Small hyaluronan oligosaccharides induce inflammation by engaging both toll-like- 4 and CD44 receptors in human chondrocytes," Biochemical Pharmacology, vol. 80, no. 4, pp. 480-490, 2010.

[22] A. L. Khoo, L. Y. A. Chai, H. J. P. M. Koenen et al., "Regulation of cytokine responses by seasonality of vitamin D status in healthy individuals," Clinical and Experimental Immunology, vol. 164, no. 1, pp. 72-79, 2011.

[23] J. S. Adams, S. Ren, P. T. Liu et al., "Vitamin D-directed rheostatic regulation of monocyte antibacterial responses," Journal of Immunology, vol. 182, no. 7, pp. 4289-4295, 2009.

[24] P. T. Liu, S. Stenger, H. Li et al., "Toll-like receptor triggering of a vitamin D-mediated human antimicrobial response," Science, vol. 311, no. 5768, pp. 1770-1773, 2006.

[25] X. Lu, P. Farmer, J. Rubin, and M. S. Nanes, "Integration of the $\mathrm{Nf} \kappa \mathrm{B}$ p65 subunit into the vitamin D receptor transcriptional 
complex: identification of p65 domains that inhibit 1,25dihydroxyvitamin D3-stimulated transcription," Journal of Cellular Biochemistry, vol. 92, no. 4, pp. 833-848, 2004.

[26] P. K. Farmer, X. He, M. L. Schmitz et al., "Inhibitory effect of NF-kappaB on 1,25-dihydroxyvitamin $\mathrm{D}(3)$ and retinoid X receptor function," American Journal of Physiology. Endocrinology and Metabolism, vol. 279, no. 1, pp. E213-E220, 2000.

[27] S. Wu, A. P. Liao, Y. Xia et al., "Vitamin D receptor negatively regulates bacterial-stimulated NF- $\kappa \mathrm{B}$ activity in intestine," American Journal of Pathology, vol. 177, no. 2, pp. 686-697, 2010.

[28] L. C. Tetlow and D. E. Woolley, "Expression of vitamin D receptors and matrix metalloproteinases in osteoarthritic cartilage and human articular chondrocytes in vitro," Osteoarthritis and Cartilage, vol. 9, no. 5, pp. 423-431, 2001.

[29] B. N. R. Lee, T. H. Kim, J. B. Jun et al., "Upregulation of interleukin- $1 \beta$ production by 1,25 -Dihydroxyvitamin $\mathrm{D} 3$ in activated human macrophages," Molecular Biology Reports, vol. 38, no. 3, pp. 2193-2201, 2011.

[30] J. E. Do, S. Y. Kwon, S. Park, and E. S. Lee, "Effects of vitamin $\mathrm{D}$ on expression of Toll-like receptors of monocytes from patients with Behçet's disease," Rheumatology, vol. 47, no. 6, pp. 840-848, 2008.

[31] L. C. Tetlow and D. E. Wooley, "The effects of $1 \alpha, 25-$ dihydroxyvitamin D3 on matrix metalloproteinase and prostaglandin E2 production by cells of the rheumatoid lesion," Arthritis Research, vol. 1, no. 1, pp. 63-70, 1999.

[32] V. Gambhir, J. Kim, S. Siddiqui et al., "Influence of 1,25dihydroxy vitamin D3 on TLR4-induced activation of antigen presenting cells is dependent on the order of receptor engagement," Immunobiology, vol. 216, no. 9, pp. 988-996, 2011.

[33] R. Lin, N. Amizuka, T. Sasaki et al., " $1 \alpha, 25$-dihydroxyvitamin D3 promotes vascularization of the chondro-osseous junction by stimulating expression of vascular endothelial growth factor and matrix metalloproteinase 9," Journal of Bone and Mineral Research, vol. 17, no. 9, pp. 1604-1612, 2002.

[34] L. C. Tetlow and D. E. Woolley, "Effects of $1 \alpha, 25$-dihydroxyvitaminD3 on matrix metalloproteinase expression by rheumatoid synovial cells and articular chondrocytes in vitro," Annals of the New York Academy of Sciences, vol. 878, pp. 615$618,1999$.

[35] L. Haywood, D. F. McWilliams, C. I. Pearson et al., "Inflammation and angiogenesis in osteoarthritis," Arthritis and Rheumatism, vol. 48, no. 8, pp. 2173-2177, 2003.

[36] C. A. Peterson and M. E. Heffernan, "Serum tumor necrosis factor-alpha concentrations are negatively correlated with serum $25(\mathrm{OH}) \mathrm{D}$ concentrations in healthy women," Journal of Inflammation, vol. 5, p. 10, 2008. 


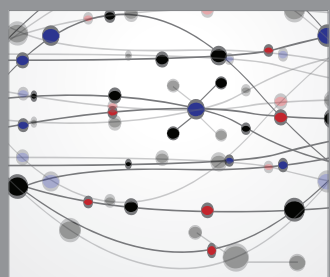

The Scientific World Journal
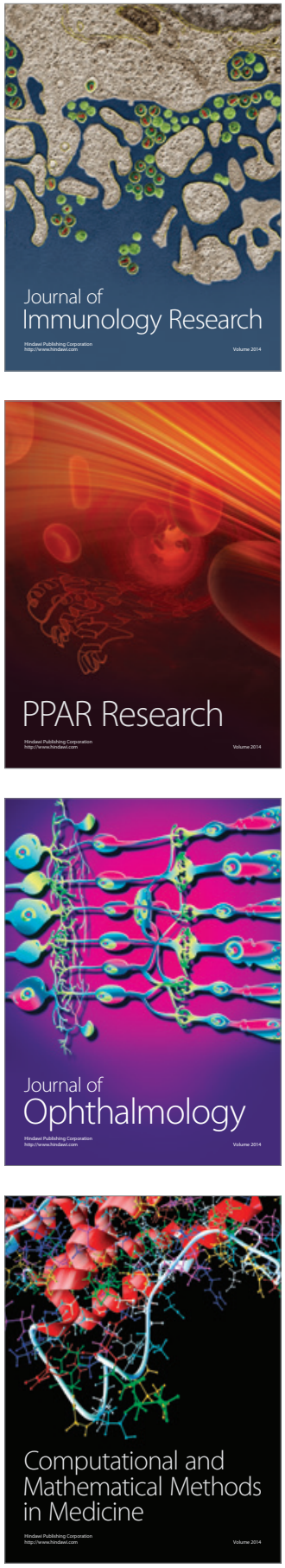

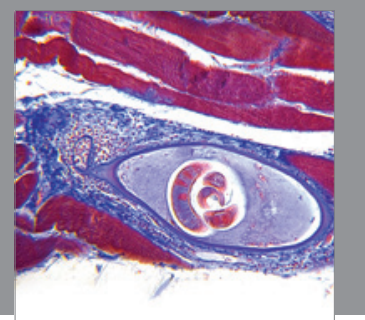

Gastroenterology

Research and Practice
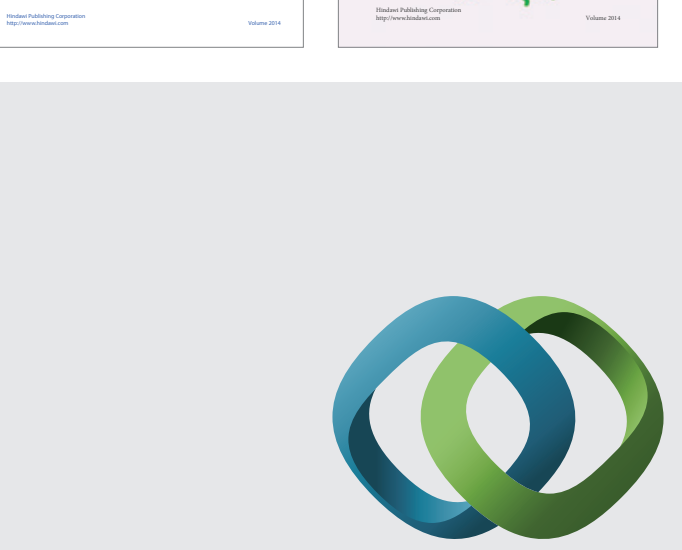

\section{Hindawi}

Submit your manuscripts at

http://www.hindawi.com
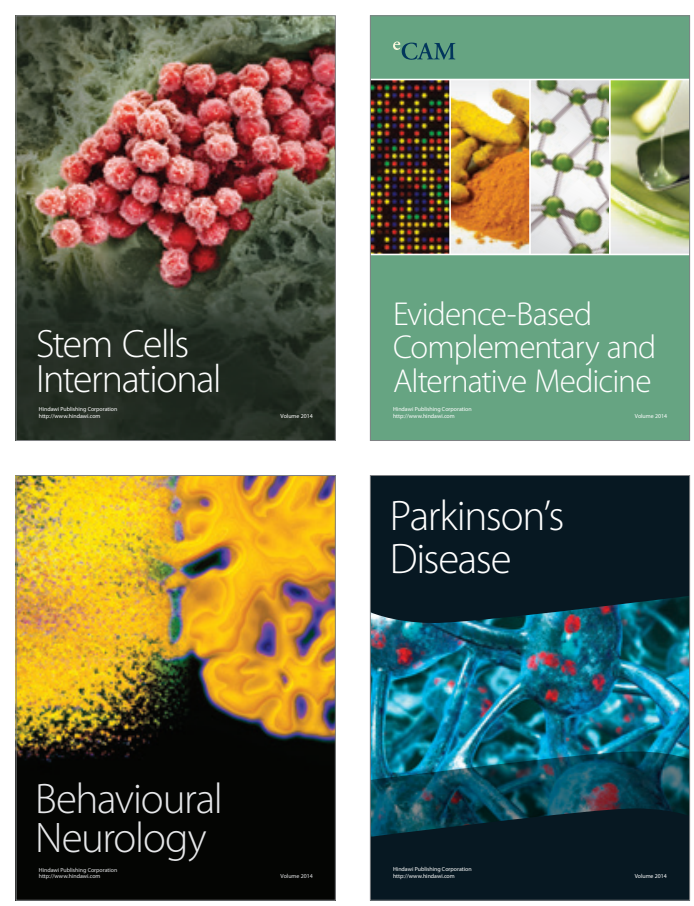

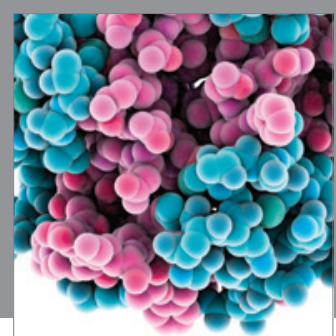

Journal of
Diabetes Research

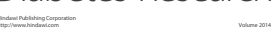

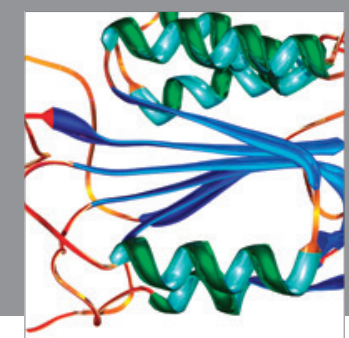

Disease Markers
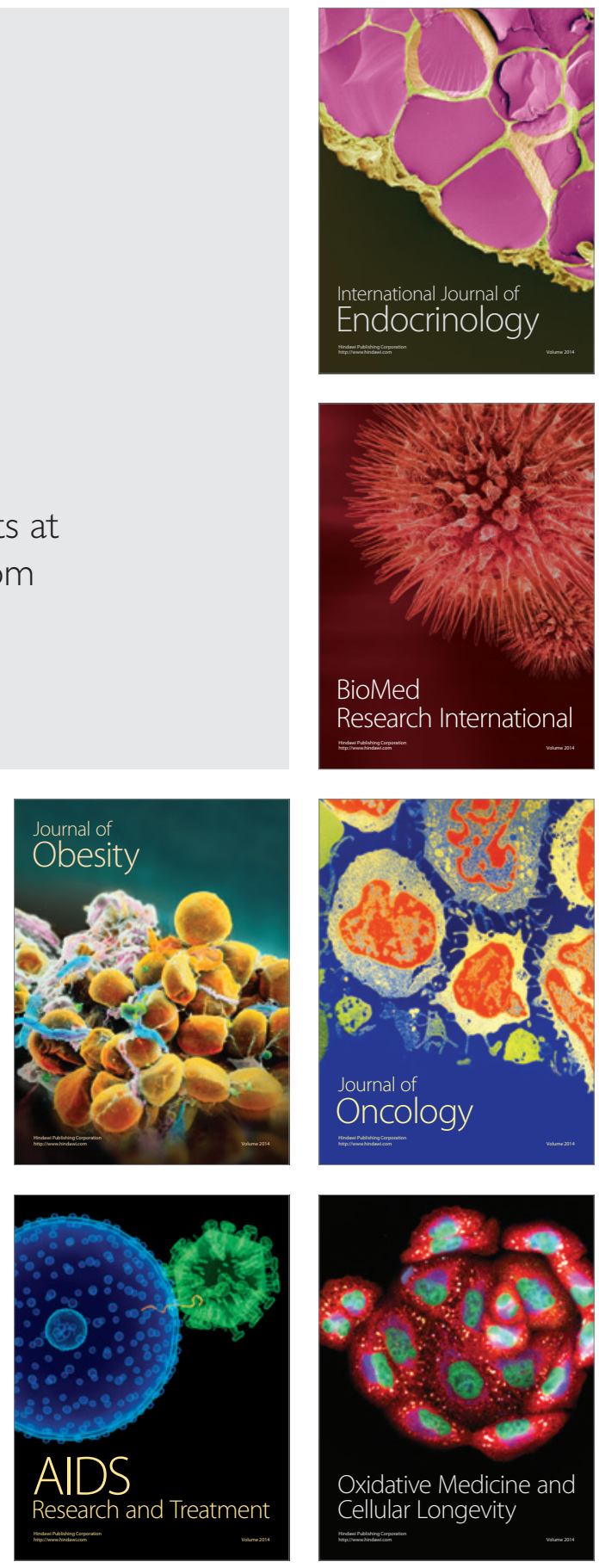